\title{
Do We Currently Have Guidelines to Select an Adequate Prosthetic Valve to Surgically Treat Active Infective Endocarditis?
}

Pascal M. Dohmen

To view enhanced content go to www.cardiologytherapy-open.com

Received: December 6, 2013 / Published online: January 23, 2014

(c) The Author(s) 2014. This article is published with open access at Springerlink.com

\section{ABSTRACT}

Active infective endocarditis is a devastating disease with high morbidity and mortality, which generally needs surgery and appropriate antimicrobial therapy. Selecting an adequate valve prosthesis is a central issue during surgery. This editorial discusses the recent literature available on this important topic.

Keywords: Active infective endocarditis; Surgery; Prosthetic valve endocarditis

\section{SURGICAL OPTIONS}

Active infective endocarditis (AIE) is a devastating disease with high morbidity and mortality. In general, surgery is a fundamental part of AIE therapy along with administering an appropriate antimicrobial therapy. For decades,

P. M. Dohmen $(\varangle)$

Department of Cardiac Surgery, Heart Center Leipzig, University of Leipzig, Leipzig, Germany

e-mail: pascal.dohmen@yahoo.de

P. M. Dohmen

Department of Cardiothoracic Surgery, University of the Free State, Bloemfontein, South Africa morbidity and mortality in surgically treated AIE has not changed, although surgical methods have steadily become more sophisticated. Several risk factors to predict hospital mortality have been identified. In general, patient morbidity and mortality depends on the specific type of microbe involved and the impact on tissue destruction, multiple resistances to antibiotic therapy, and the timing of appropriate antibiotic therapy administration. Clear differences need to be made between native and prosthetic valve AIE, since mortality in the latter group is much higher compared with native valve AIE. This is due to the formation of biofilms in prosthetic heart valves, which are more resistant against antimicrobial therapy and therefore more easy to develop. Healthy native valves, however, have an intact endothelium which prohibits the development of biofilms. Finally, the existence of previous embolisms, either central or peripheral, will have a significant impact on patient outcome. Our group found in a multivariate analysis that female gender, sepsis with cardiogenic shock, and preoperative embolisms are predictors for hospital mortality [1]. Surgical therapy options to treat AIE are similar to those used in the 
treatment of valve degeneration. The underlying principle is to limit foreigner tissue or material as much as possible in AIE surgical treatment. Therefore, the primary goal during AIE surgical valve therapy is to perform valve-sparing procedures. Preservation of the involved valve is crucial and generally possible if AIE is limited to the valve leaflets. Different reconstruction techniques are available, such as partial or total leaflet excision with leaflet reconstruction, followed by the use of an allo- or xenogenic patch material. Therefore, complete excision of the macroscopically visualized infected tissue needs to be carried out. This will overcome the persistence of AIE, which eventually could contaminate the foreigner material used during surgery, leading to the development of a new biofilm. If the valve annulus or the infection is extended to the surrounding tissue, then valve replacement needs to be considered. In extremely severe cases where extensive cardiac tissue is involved in combination with multivalve involvement, and there is no longer a possibility to fix a heart valve prosthesis adequately, then cardiac transplantation is the only option left. This therapy option, however, needs to be considered very carefully, since immunosuppressive medication needs to be administered in primary septic patients. Also, the timing of therapy is difficult, and until an appropriate organ is available for the patient, bridging is required. For all AIE treatment, the key point to treatment is that this specific disease needs to be treated interdisciplinary. Adequate antibiotic therapy should support the surgical treatment, of which the effectiveness can be reflected by the control of clinical parameters, using echocardiographic follow-up, blood cultures, and laboratory examination.

For the treatment of sepsis we know "the Tarragona strategy", which means "Hit hard and early", with an appropriate antimicrobial therapy is a crucial treatment strategy. This should also be the strategy in the surgical treatment of AIE, by early and complete debridement of the infective valve tissue, eventually including the surrounding tissue. But this raises the question "What will be the adequate valve prosthesis in AIE?". In general, heart valve prostheses can be divided into mechanical and biological origin, and the choice will be based on age, life expectancy, co-morbidities and compliance with anticoagulation. The only available guidelines for appropriate selection of valve prosthesis for the surgical treatment of AIE are performed by Byrne et al. [2]. Unfortunately, the information given on accurate elected valve prosthesis is not specific for AIE treatment, although the same criteria for valve replacement due to degeneration are used. Again the choice of prosthesis was recommend to be based on a patient's age, life expectancy, co-morbidities and compliance with anticoagulation. This important recommendation in choosing the correct and most optimal valve prosthesis is supported on Class IIa, level of evidence B or Class IIb, level of evidence B analyses [1]. When evaluating the studies on which these recommendations are based, it is important to know that the most recent paper was published in 2002 [1]. Also, the patient cohorts are on average only a limited number, between a minimum of 41 and a maximum of 221 patients. This makes it difficult to find statistically significant differences between groups. In the case of a patient suffering from either native valve or prosthetic valve endocarditis with periannular abscess, it has been indicated that the use of a homograft may be reasonable [1]. Today, it is well accepted that homografts have the most resistance against infection. This has been demonstrated in vascular prosthetic graft infection, where using 
a vascular allograft implantation leads to the highest cure rates of prosthetic infections [3]. In general, in cardiac surgery, younger patients receive mechanical heart valves and older patients get a stented or stentless bioprosthesis implanted. The highly diseased younger patients receive cryopreserved allograft. The reason for this is that allografts are rare and therefore generally reserved for younger patients. It would be of great interest to get randomized studies with narrow confidence interval or systemic reviews of RCTs to investigate the impact of different available valve prosthesis in the clinical outcome of patients.

\section{COMPARING DIFFERENT VALVE PROSTHESES}

It is difficult to compare different valve prostheses used during AIE since different patient populations usually receive different valve prostheses; for example, in my practice I prefer the use of cryopreserved allografts for the most severe cases. Following cardiopulmonary bypass, the time to allograft should be as short as possible to reduce additional complications. Therefore, one needs to determine the correct valve annulus prior to surgery as the thawing procedure of cryopreserved allografts is time consuming and delicate. Patients with less complicated AIE receive a stented biological heart valve and in the cases where no cryopreserved allograft is available, a stentless bioprosthesis is used. In general, stented biological heart valve prostheses have a good prognosis; however, these patients generally suffer from localized AIE without embolism. Patients receiving a stentless valve are at higher risk of mortality and morbidity in comparison, although these patients usually have a more extended diseased endocarditis compared to patients who receive mechanical valve prostheses. Therefore, it is difficult to make comparisons between the different valve prostheses. Differences in patient age and valve recommendation also make it difficult to compare the different valve prostheses as younger patients, who are at a lower risk of mortality compared to older patients due to age, are more likely to receive a mechanical valve rather than older patients. Personally I do not favour the use of a mechanical heart valve even in young patients with AIE if cerebral embolization is diagnosed, as a fulminate bleeding during permanent anticoagulation can occur. It is an extremely difficult and complex disease and treatment needs to be individually planned for each patient taking into consideration the advantages and disadvantages. Delahaye performed a systemic review about the benefit of early surgery in infective endocarditis [4]. The analysis of the literature suggests that early surgery is favorable for long-term survival; however, cardiologist does also see many patients, especially with native valve endocarditis that can be cured without surgery. This is of course difficult for a surgeon to mention, but it shows again the complexity of this devastating disease that always needs an interdisciplinary team to make the correct decision for each individual patient. If surgery is needed, guidelines are available for the optimal timing [5].

Since the morbidity and mortality has not been changed over decades in AIE surgery, RCTs in combination with real world data could be a point of interest to decrease these rates. Every effort needs to be undertaken to reduce high morbidity and mortality due to AIE.

\section{ACKNOWLEDGMENTS}

No funding or sponsorship was received for this study or publication of this article. Pascal Dohmen 
meets the ICMJE criteria for authorship for this manuscript, takes responsibility for the integrity of the work as a whole, and has given final approval for the version to be published.

Conflict of interest. The author has no conflicts of interest to disclose.

Compliance with ethics guidelines. This article does not contain any new studies with human or animal subjects performed by the author.

Open Access. This article is distributed under the terms of the Creative Commons Attribution Noncommercial License which permits any noncommercial use, distribution, and reproduction in any medium, provided the original author(s) and the source are credited.

\section{REFERENCES}

1. Gabbieri D, Dohmen PM, Linneweber J, et al. Early outcome after surgery for active native and prosthetic aortic valve endocarditis. J Heart Valve Dis. 2008;17:508-24.

2. Byrne JG, Rezai K, Sanchez JA, et al. Surgical management of endocarditis: the Society of Thoracic Surgeons clinical practice guideline. Ann Thorac Surg. 2011;91:2012-9.

3. Moon MR. Prosthetic valve selection in patients with left-sided endocarditis: bioprosthetic or mechanical valves? Curr Opin Cardiol. 2014 (Epub ahead of print).

4. Delahaye F. Is early surgery beneficial in infective endocarditis? A systemic review. Arch Cardiovasc Dis. 2011;104:35-44.

5. Habib G, Hoen B, Tornos P, et al. Guidelines on the prevention, diagnosis, and treatment of infective endocarditis (new version 2009): the Task Force on the Prevention, Diagnosis, and Treatment of Infective Endocarditis of the European Society of Cardiology (ESC). Endorsed by the European Society of Clinical Microbiology and Infectious Diseases (ESCMID) and the International Society of Chemotherapy (ISC) for Infection and Cancer. Eur Heart J. 2009;30:2369-413. 